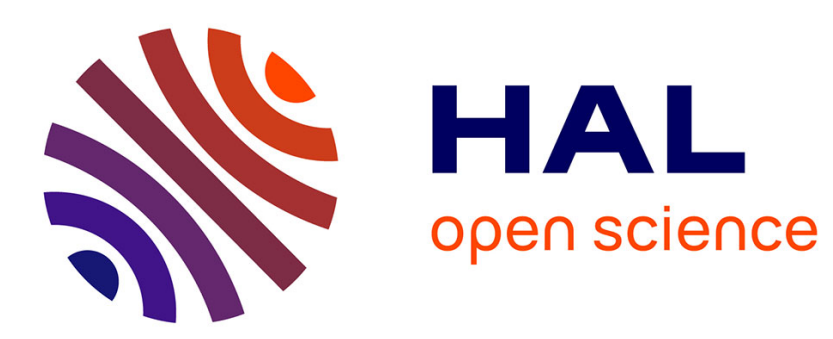

\title{
Alliance et séniorité. Le cas des Lao du nord-est de la Thaïlande
}

\author{
Bernard Formoso
}

\section{To cite this version:}

Bernard Formoso. Alliance et séniorité. Le cas des Lao du nord-est de la Thaïlande. L'Homme - Revue française d'anthropologie, 1990, 30 (115), pp.71-97. 10.3406/hom.1990.369282 . hal-01415315

\section{HAL Id: hal-01415315 \\ https://hal.science/hal-01415315}

Submitted on 16 Apr 2018

HAL is a multi-disciplinary open access archive for the deposit and dissemination of scientific research documents, whether they are published or not. The documents may come from teaching and research institutions in France or abroad, or from public or private research centers.
L'archive ouverte pluridisciplinaire HAL, est destinée au dépôt et à la diffusion de documents scientifiques de niveau recherche, publiés ou non, émanant des établissements d'enseignement et de recherche français ou étrangers, des laboratoires publics ou privés. 


\section{Alliance et séniorité Le cas des Lao du nord-est de la Thaïlande} Bernard Formoso

\section{Citer ce document / Cite this document :}

Formoso Bernard. Alliance et séniorité Le cas des Lao du nord-est de la Thaïlande. In: L'Homme, 1990, tome 30 n¹15. pp. 71-97;

doi : 10.3406/hom.1990.369282

http://www.persee.fr/doc/hom_0439-4216_1990_num_30_115_369282

Document généré le 29/03/2016 


\section{Alliance et séniorité Le cas des Lao du nord-est de la Thaillande*}

Bernard Formoso, Alliance et séniorité. Le cas des Lao du nord-est de la Thailande. L'auteur décrit le système de parenté lao en le comparant à son homologue siamois. Ce faisant il met en évidence l'importance considérable que ce système confère à la dichotomie aîné/cadet. Il replace cette dichotomie dans un contexte socio-culturel plus général et en analyse l'impact tant sur le plan terminologique que dans le registre des pratiques matrimoniales.

Jamais véritablement pris en compte comme objet d'étude, hormis quelques notes descriptives et succinctes dans le cadre de monographies villageoises, le système de parenté lao présente pourtant des traits qui le démarquent non seulement de ses homologues relevant de la même famille ethnolinguistique (branche thaï du groupe thaï-kadai), mais aussi de la plupart des autres systèmes de régime indifférencié. Il se caractérise en effet par une considérable emphase de la relation aîné-cadet et un ensemble de prohibitions matrimoniales dont le champ d'application l'inscrit dans les systèmes d'alliance semicomplexes. L'objet du présent article est triple. Dégager les propriétés du système de parenté lao en le comparant à son homologue siamois, les deux systèmes ayant évolué dans le même contexte social et politique à partir d'un tronc culturel commun ; mettre en évidence le rôle que jouent l'ordre de naissance et les écarts d'âge dans la définition normative des rapports interpersonnels au sein des sociétés lao et siamoise contemporaines ; enfin, analyser sur le plan tant théorique que pratique la manière dont le critère de l'âge structure la terminologie de parenté des Lao et régule les choix matrimoniaux au sein de cette société.

L'étude $^{1}$ se fonde sur des observations ethnographiques et des relevés statistiques effectués dans deux villages du nord-est de la Thaillande, région située sur la rive droite du Mékong et qui est plus connue sous le nom de plateau de Khorat. Ce plateau, peu fertile et soumis à un régime irrégulier de pluies (Platenius $1963: 9$ ), occupe près d'un tiers du territoire national et rassemblait en $198434,9 \%$ de la population thaillandaise, soit 17,7 millions d'habitants (NSO 
1984 : 12). A défaut d'être la plus ancienne zone de peuplement lao, le nord-est de la Thailande est la première sur le plan démographique. On peut en effet estimer le nombre de membres de l'ethnie lao qui y vivent à 15 millions contre 3,5 millions dans le nord de la Thaillande et 1,7 million (soit huit fois moins) en République démocratique populaire lao.

Appelés Khon Isan ("Gens du nord-est ») par les populations au contact desquelles ils vivent, les Lao du plateau de Khorat se nomment eux-mêmes Thai Isan ou Lao Isan selon qu'ils souhaitent affirmer leur citoyenneté thailandaise ou souligner leur origine et leur filiation culturelle lao. Immergés dans une société autre, ils sont soumis à un processus d'acculturation que depuis deux décennies la conjonction de plusieurs facteurs a amplifié : réduction des échanges commerciaux et des déplacernents entre rives droite et gauche du Mékong suite à la mise en place d'un régime socialiste au Laos, renforcement des structures d'encadrement étatique, amélioration des voies et moyens de communication, ouverture sur l'économie de marché et, corrélativement, essor de l'émigration temporaire ou définitive en direction des principaux pôles de croissance du pays. Ce processus d'acculturation n'est pas uniforme et varie suivant la position géographique et le niveau de développement des villages du nord-est. Son impact inévitable sur les systèmes de classification et l'organisation sociale des Isan justifie à lui seul la mise en perspective que l'on propose ici des systèmes de parenté lao et siamois.

Quoique localisés dans la même province de Khon Kaen, les deux villages étudiés présentent de nombreuses différences. L'un, appelé Ban Amphawan, est situé à $13 \mathrm{~km}$ seulement de la ville de Khon Kaen, capitale de la province du même nom et pôle administratif régional. Ban Amphawan, fondé en 1918, comptait en 1985773 habitants répartis en 117 maisonnées (6 personnes en moyenne par maisonnée). Ce village, de petite taille, se trouve à l'intérieur du périmètre irrigué de Nong Waï et grâce aux facilités d'approvisionnement en eau que lui offre une telle infrastructure, il obtient deux récoltes annuelles de riz, l'une en saison sèche et l'autre à la saison des pluies ; les agriculteurs pratiquent en outre des cultures intensives de maraîchage, diverses formes d'élevage, notamment la pisciculture. L'autre localité, Ban Han, est beaucoup plus éloignée de la ville puisqu'elle est située à près de $80 \mathrm{~km}$ de Khon Kaen. Fondé en 1907, ce village est plus important : il comptait en 19851119 habitants et 214 maisonnées (soit comme à Ban Amphawan 6 personnes en moyenne par maisonnée). Tributaire des pluies pour leur production de base - le riz - les habitants n'obtiennent qu'une récolte annuelle et complètent leurs revenus agricoles en élevant du bétail et en pratiquant diverses cultures sèches dont le manioc, le kenaf (Hibiscus cannabinus L.) et la canne à sucre. 


\section{TRAITS GÉNÉRAUX DU SYSTÈME DE PARENTÉ LAO}

Le système de parenté lao et son homologue siamois sont similaires à bien des égards. De type indifférencié, ils présentent cependant une inflexion matrilinéaire se manifestant notamment par un mode de transmission des biens qui, tout en étant bilatéral, tend à privilégier les filles aux dépens des garçons, ainsi que dans l'uxorilocalité initiale de la majorité des couples mariés (De Young 1955 : 64 ; Condominas 1961 : 1 ; Wijeyewardene 1967 : 68-69 ; Lux 1969 : 3 ; Kaufman $1976: 29$ ). Comme le remarque Condominas (ibid. : 1) à propos des Lao de la rive gauche du Mékong, cette matrifocalité, loin d'être révélée par le respect systématique de règles, s'exprime plutôt sous forme de régularités statistiques et les principes idéalement définis doivent dans la pratique s'accommoder de multiples exceptions. Chez les Lao Isan du nord-est et les Lao Yüan (ou Khon Müang) du nord de la Thaïlande, cette inflexion est également marquée sur le plan religieux par la croyance en un esprit maternel nommé phi: sù: $a$ (« esprit du lignage ») dans le premier cas et phi: pu: ja: (« esprit des frères et sœurs aînés des grands-parents ») dans le second. Un tel esprit n'est pas identifié à un ancêtre connu et de ce fait n'occupe pas une position structurale précise. Comme l'écrit Turton (1972 : 237), il présente la triple caractéristique d'être anonyme, divisible à l'infini et invisible. Les groupes matrilinéaires formés autour du culte de cet esprit sont d'une profondeur généalogique variable (entre 3 et 8 générations). Les Isan les appellent sù:a phi:, les Yüan kôk phi: (notions que la plupart des auteurs traduisent par « clan » ou « lignage de l'esprit »). Si les hommes sont en principe rattachés au groupe de leur mère, une grande flexibilité rend possible leur intégration à celui de l'épouse (Turton 1972: 221 ; Cohen \& Wijeyewardene 1984 : 250). Ces groupes, que l'on pourrait plus adéquatement désigner par le terme ramage ${ }^{2}$, ne constituent pas des corporate groups au sens strict de l'expression, ainsi que le note Davis (1984 : 54) à propos des Yüan. En effet ils ne sont pas régis par un principe d'endogamie ou d'exogamie, ils ne déterminent pas le statut juridique et politique des individus, ne jouent aucun rôle dans la sphère des activités productives, ne prêtent à aucune solidarité économique particulière et enfin ne sont pas fondés sur la propriété collective et indivis de biens (hormis celle d'un sanctuaire dans le cas des Yüan).

Ces groupes ne prennent corps en fait qu'à l'occasion de rites célébrés en l'honneur du génie tutélaire. Précisons qu'une distinction s'impose à ce niveau entre le culte que les Isan rendent au phi: sù: $a$ et celui que les Yüan rendent au phi: pu: ja: En effet, alors que dans le premier cas les rites n'ont lieu que lorsqu'il y a suspicion de maladie ou de malheur causé par l'esprit en représailles de la transgression d'un tabou (sexuel notamment) par ses descendants ou leur conjoint, chez les Yüan, en plus de ces rites occasionnels, d'autres sont organisés à date fixe chaque année, ou pour les plus importants, tous les trois ans (Turton 1972 : 246 ; Davis 1984 : 55). De plus, alors que chez les Isan l'esprit 
n'est pas localisé dans l'espace, il possède un petit sanctuaire dans le cas des Yüan. Enfin, si chez ces derniers un ancien, généralement une femme âgée, est titulaire de la fonction honorifique de médiateur entre le groupe de filiation et l'ancêtre fondateur (Turton 1972 : 223), chez les Isan la charge de maître de cérémonie peut être assumée selon les circonstances par n'importe quel membre du groupe. Présentant à quelques nuances près les mêmes propriétés, les cultes que les Yüan et les Isan rendent à leur ancêtre maternel ont suivi la même évolution : ces dernières décennies ils ont en effet subi un très net déclin qui, selon Cohen et Wijeyewardene (1984: 249) résulterait de l'affaiblissement de l'autorité des anciens et de l'influence croissante des valeurs occidentales, notamment en matière de sexualité. Mougne (1984 : 305) a montré par ailleurs que le degré de participation au culte de l'esprit maternel diminue au fur et à mesure qu'augmentent l'émigration féminine, le niveau de revenus et le degré d'instruction. Davis (1984 : 55) suggère comme autre cause possible l'introduction de noms patronymiques par le gouvernement thailandais. Ce déclin paraît plus ancien et plus marqué chez les Isan (Mizuno 1971 : 105), et alors que dans le nord de la Thaïlande un accroissement du nombre de femmes médium est venu compenser, semble-t-il, la désaffection des Yüan pour le culte de l'esprit maternel ancestral (Irvine 1984 : 315), une telle substitution n'est pas observée chez les Lao du nord-est.

Bien que les idylles romantiques soient valorisées par les Lao et les Siamois, et que les choix matrimoniaux laissent une grande part aux initiatives des jeunes gens (Lux 1969 : 3 ; Mizuno 1971 : 84 ; Keyes 1975 : 289), des paramètres politiques ou socio-économiques opèrent pour définir dans certains cas des stratégies d'alliance. Si au sein de l'aristocratie et des élites urbaines l'hypergamie et l'hypogamie sont courantes, en milieu rural la tendance dominante est aux mariages homogames : on épouse des personnes sensiblement de même condition. C'est lorsqu'il y a disparité économique au profit du côté de l'époux que le choix d'un mode de résidence virilocal intervient le plus souvent (Kemp 1970 : 83 ; Keyes 1975 : 287). Au niveau des communautés villageoises, les tendances matrimoniales varient en fonction de facteurs économiques, démographiques, historiques et géographiques. Contrairement aux assertions que certains auteurs (notamment Doré 1987 : 288) fondent sur un petit nombre de matériaux ethnographiques et qui fixent comme règle générale l'endogamie villageoise par l'intermédiaire du mariage préférentiel des cousins croisés et parallèles, certaines communautés présentent à un moment donné un fort taux de mariages endogames et quelques années ou générations plus tard la tendance inverse (Kemp 1983 : 32). Ainsi, alors qu'à Ban Han la proportion d'hommes mariés originaires du village était en 1969 de 54,8\%, elle avait chuté à 41,6\% quinze ans plus tard.

Bien que la polygamie ait jadis eu cours dans l'aristocratie, les Thaï et les Lao de l'époque contemporaine sont monogames dans leur immense majorité. Malgré cette monogamie, la notion de fidélité conjugale n'est pas très développée chez les hommes comme en témoigne la tradition solidement ancrée des 
mi:a no:j (« petites épouses »). A défaut d'être légitimée par les femmes, cette tradition est tolérée et les cas de divorce ou de séparation pour cause d'adultère sont assez rares. A Ban Amphawan et Ban Han par exemple, ce type de rupture n'avait affecté respectivement que $11 \%$ et $6 \%$ des unions recensées lors de l'étude. L'intégration de nouveaux membres à la maisonnée par les voies de l'adoption est assez fréquente chez les Lao (Keyes 1975 : 282-283 ; Barber $1979: 310-311)$; elle concerne surtout des enfants abandonnés ou des orphelins appartenant au même groupe de parenté.

Chez les Lao, qu'ils vivent sur les rives droite ou gauche du Mékong, ainsi que chez les Siamois, l'unité productive de base est formée d'un ménage auquel s'agrègent au cours du cycle de vie un ou plusieurs couples d'enfants mariés ${ }^{3}$. Ce groupe de parents consanguins vit soit sous le même toit, soit dans le même enclos ; selon les auteurs, il est appelé multi-household compound (Mizuno 1971 : 242), uxori-parentilocal extended family (Keyes 1975 : 287) ou matrilocal extended stem family (Potter 1976 : 151). Les liens qu'il tisse avec le voisinage sont très souvent plus denses que ceux qui l'unissent aux autres membres de la parentèle lorsque celle-ci est dispersée. Dans le cas des villages qui se signalent par un fort taux d'endogamie, relations de voisinage et de parenté tendent bien sûr à se confondre. Comme c'est généralement le cas dans les systèmes de parenté indifférenciés, les groupes conjoncturels qui se forment dans le cadre des activités de production ou à l'occasion des fêtes familiales sont la plupart du temps egocentrés, c'est-à-dire qu'ils se constituent autour d'un individu ou d'un ménage et non par référence à un groupe de parenté plus large qui pourrait être assimilé à un lignage. Ces groupes ne sont pas exclusivement fondés sur la parenté, mais intègrent aussi des voisins.

\section{LA RELATION AÎNÉ-CADET}

De tous les critères distinctifs selon lesquels se décline la position sociale de l'individu au sein de l'univers hiérarchisé que forment les sociétés lao et siamoise, l'ordre de naissance est indéniablement le plus important. Si, comme le critère du sexe, il est fondé sur une réalité biologique et ordonne d'emblée l'ensemble de la population, il présente cependant la double propriété d'être relatif et réversible. En effet, alors que l'identité sexuelle est immuable et indissociable de l'âme dans le cycle métaphysique des transmigrations auquel croient les bouddhistes lao et thaï, l'individu est toujours à la fois plus jeune et plus âgé que d'autres, même si la séniorité et le respect qu'elle procure croissent au fil des ans. La relation aîné-cadet et le code de conduite qui lui est attaché sont donc d'autant plus profondément enracinés dans les mentalités qu'ils sont quasi systématiquement marqués selon la situation sociale et que tout individu est tenu d'adopter alternativement les comportements correspondant à l'une ou l'autre de ces positions.

Les notions de patronage et de subordination sont les mieux à même de 
rendre compte du type de comportement respectivement impartis aux anciens et aux jeunes, aux aînés et aux cadets. Ainsi l'attitude des seconds envers les premiers est dictée dans l'idéal par trois sentiments : ka:orôp (le respect), chù:a fang (l'obéissance) et kre:ngcai (la crainte de blesser l'amour propre d'un supérieur) (Rabibhadana 1975 : 108). En échange, le plus âgé doit protéger (kumkhro:ng) et aider (chwajlù:a) les plus jeunes. La réciprocité qui cimente la relation entre les âges et les générations et par extension toute interaction entre supérieur et subordonné est rendue en lao et en thaï par différentes notions : bunkhun, khwam metta: karuna et katanju katawathi: ${ }^{4}$ qui toutes expriment le sentiment de gratitude auquel est tenu le récipiendaire d'une faveur (Mizuno 1971 : 224 ; Rabibhadana 1975 : 108).

Ces principes vont bien au delà d'un simple contrat moral. Transposés dans de multiples secteurs de la vie sociale, ils ne se réduisent pas à des notions et des règles explicites, utilisées à des fins tactiques. Ils imprègnent gestes et postures, conditionnent le ton, le registre lexical et les formules de politesse, ainsi que le contenu des propos échangés. Ils forment un code de conduite informel, selon l'expression de Terwiel (1984:23), un ensemble de dispositions inculquées au cours d'un processus d'apprentissage qui, au fil des situations à la fois récurrentes et diversifiées, précise les nuances du protocole à observer selon l'interlocuteur tout en renforçant l'intériorisation du schéma à la base de la relation.

La relation filiale parent/enfants, prépondérante dans la socialisation progressive de l'individu, est aussi celle où ces principes d'interaction sont exprimés avec le plus de force. En retour de l'instruction, du soutien matériel et de l'amour qu'ils reçoivent durant leur croissance, les enfants doivent témoigner à leurs parents respect et obéissance, de même qu'ils doivent éviter de leur faire perdre la face en portant publiquement atteinte à leur piep, notion métaphysique qui selon le contexte peut être traduite par " patrimoine vital » ou " prestige » (Doré 1972 : 26-30). De plus la tradition veut qu'ils expriment leur gratitude envers ceux qui les ont mis au monde et les ont élevés, non seulement en apportant à ces derniers un soutien matériel et moral au terme de leur vie, mais aussi en réalisant avant et après leur mort des mérites religieux (bun) qui leur garantiront un destin (kamwen) meilleur dans le cadre des transmigrations futures. La réciprocité typique des relations filiales couvre donc à la fois la dimension matérielle et spirituelle de la vie et se perpétue dans les rapports qui lient les vivants aux morts, les ancêtres à leurs descendants.

Comme le prouve l'usage extensif que les Lao font du vocabulaire de la parenté, le code de conduite idéal qui unit parents et enfants, mais aussi grands-parents et petits-enfants ou germains aînés et cadets sert de modèle à l'idéologie pour définir, dans un contexte social plus large, le contenu et la tonalité des rapports entre personnes d'âge et de statut différents. La relation patron-employés est ainsi posée pour partie dans les termes d'un écart générationnel qui conforte l'autorité et la respectabilité du patron, appelé tao kae: (" ancien-âgé ») $)^{5}$ et pour partie dans les termes d'une relation filiale qui soustend affection, confiance et loyauté réciproques, les employés étant qualifiés de 
lu:k no:ng (« enfant-germain cadet »). D'autre part l'usage courant qui est fait de la terminologie d'adresse ou de référence préfère à la spécification des positions généalogiques réelles un mode de classement générationnel très simplifié, qui assimile les personnes apparentées ou non aux consanguins les plus proches. Ego identifie ainsi suivant leur âge les personnes qui lui sont familières, notamment les habitants de son village, à ses grands-parents, parents, frères et sœurs, enfants ou petits-enfants.

Le recours à un tel mode de classification a plusieurs effets. En toutes circonstances il signifie à l'individu son ordre de naissance relatif et le type de comportement qu'une telle position suppose. Comme le remarque Kemp (1984 : $60)$, il place les relations sociales dans un contexte d'amour, de loyauté et d'absence de calcul qui caractérise l'idéologie sinon toujours la pratique de la parenté. Enfin, au niveau villageois, il rappelle à l'individu qu'il appartient à un ensemble organique et hiérarchisé, appartenance que symbolise de manière plus ponctuelle le culte collectif rendu à l'esprit fondateur et protecteur de la communauté, génie tutélaire que les Isan appellent ta: pu: ba:n (« grand-père maternel et paternel du village $»)$.

Les manipulations symboliques auxquelles prêtent les termes de parenté n'interviennent que lorsque les partenaires sociaux sont animés par le souci mutuel d'imprimer à leur relation une tonalité affective et une inflexion normative propices à l'établissement d'une coopération étroite et durable. Lorsqu'une telle motivation n'est pas partagée, l'inégalité de statut est exprimée sur le plan métaphorique par des oppositions telles que su:ng/tam (« haut/bas ») et jai/no:j («grand/petit ») ou bien encore par un système formel et très complexe de rangs, grades et titres honorifiques.

Le code de conduite informel, de mise entre personnes d'âge et plus généralement de statuts différents, est plus ou moins strictement respecté selon la qualité des rapports qu'entretiennent ces personnes. Il opère cependant à des degrés divers dans la plupart des registres relationnels des sociétés lao et thaï contemporaines. Caractéristique des rapports de clientèle structuralement prépondérants au sein de ces sociétés, il prédomine dans les divers secteurs de l'activité économique (Korff 1983, 1986 ; Kuwinpant 1984), de même qu'il structure les rapports entre supérieurs et subordonnés tant à l'armée que dans la fonction publique (Riggs 1966 ; Siffin 1966). Il est d'autre part à la base des rapports entre sacré et profane, bonzes et laïques. Il est à l'œuvre à l'intérieur du sangha, la communauté monastique, à travers un ensemble de grades liés à l'âge, à l'ancienneté dans les ordres ou aux diplômes religieux. Dans le domaine des croyances pré-bouddhistes il situe les êtres humains dans les étages intermédiaires d'un univers cosmique peuplé de multiples divinités. Enfin, lorsqu'un transfert de savoir ou de pouvoirs métaphysiques est en jeu, il transparaît dans la relation 'a:ca:n/lu:ksit (« professeur, maître/élève, disciple »). 


\section{TERMINOLOGIE DE RÉFÉRENCE ET BRANCHES COLLATÉRALES}

Marquée sur le plan lexical par une influence thaï, la terminologie de parenté isan n'en reste pas moins caractérisée par un vocabulaire de base lao (tabl. 1). $\check{A} \mathrm{G}+2$ et au-dessus, cette terminologie, comme ses homologues siamois et lao, distingue entre patri- et matrilatéraux (fig. 1). La même dichotomie est reprise à $G+1$ uniquement dans le cas des cadets, le principe de séniorité étant alors défini non par rapport à Ego, mais suivant l'ordre de naissance des collatéraux de la génération immédiatement supérieure. Selon ce principe les oncles et les tantes sont distingués du père (pho:) et de la mère (mae:) par les termes lung $(\mathrm{FB}+, \mathrm{MB}+), p a:(\mathrm{FZ}+, \mathrm{MZ}+), n a:(\mathrm{MZ}-$, $\mathrm{MB}-$ ), ' $a: w(\mathrm{FB}-)$ et ' $a$ : $(\mathrm{FZ}-)$. Si à $\mathrm{G}+1$ le critère du sexe opère dans le cas des collatéraux aînés, il est secondaire au niveau des cadets sauf, et c'est là une propriété des systèmes lao et isan, dans le cas des patrilatéraux. On note par ailleurs que la terminologie n'établit aucune distinction entre cousins croisés et parallèles.

Une autre propriété que partagent les nomenclatures de parenté lao et isan et qui les démarque du système classificatoire des Siamois, tient au fait que le champ d'application des termes employés pour désigner les germains du père ou de la mère s'étend vers le bas en vertu non pas d'une règle de projection oblique réduite à certaines positions structurales comme dans le cas des systèmes crow-omaha étudiés par Lounsbury (1964), mais à l'inverse selon une règle de projection verticale et généralisée. En effet, Ego désigne d'un même terme ses oncles ânés et leurs fils ou petits-fils, de même qu'il assimile ses tantes et leurs descendantes, ses oncles cadets patri- ou matrilatéraux et leurs enfants ou petits-enfants de sexe masculin, etc. L'équivalence formelle ainsi établie entre consanguins de générations différentes permet de diviser la parentèle en branches collatérales appelées sù: $a^{6}$ et qui sont globalement définies par la position structurale qu'occupe leur fondateur par rapport aux géniteurs d'Ego.

Précisons que le mécanisme qui fait dériver des ascendants de la génération immédiatement supérieure le statut relatif des cousins n'est pas l'apanage exclusif de la terminologie de parenté lao. Un tel mécanisme opère également dans la nomenclature siamoise à deux nuances près : il joue là uniquement pour les cousins au premier degré ; le déplacement des termes de parenté d'une position structurale vers une autre s'effectue alors non plus par translation verticale, mais selon un mouvement horizontal à l'intérieur d'un même niveau générationnel, les cousins d'Ego étant assimilés à ses frères et sœurs aîné(e)s (phi:) ou cadet(te)s (no:ng) selon qu'ils descendent des germains aînés ou cadets de ses propres parents (fig. 2). Chez les Siamois l'absence de profondeur généalogique conférée à la règle de projection interdit donc toute structuration de la parentèle en branches collatérales.

Des différents critères qui déterminent la position structurale des individus 


\begin{tabular}{|c|c|c|}
\hline GÉNÉRATION & DENOTATA & DÉSIGNATIONS/VARIANTES \\
\hline$G+3$ & $\begin{array}{l}\text { FFF, FFFB } \\
\text { FFM, FFFZ } \\
\text { MFF, MFFB } \\
\text { MFM, MFFZ }\end{array}$ & $\begin{array}{l}\text { pu: thwat/pu: tha:o/pu: jai } \\
\text { ja: thwat/ja: tha:o/ja: jai } \\
\text { pho: sô:n/pho: jai tha:o } \\
\text { mae: sô:n/mae: jai tha:o }\end{array}$ \\
\hline$G+2$ & $\begin{array}{l}\text { FF, FFB, FFZH } \\
\text { FM, FFZ, FFBW } \\
\text { MF, MFB, MFZH } \\
\text { MM, MMZ, MMBW }\end{array}$ & $\begin{array}{l}\text { pu:/pho: pu: } \\
\text { ja:/mae: ja: } \\
\text { ta:/pho: jai/pho: sô:n } \\
\text { ja:j/mae: jai/mae: sô:n }\end{array}$ \\
\hline$G+1$ & $\begin{array}{l}\mathrm{F} \\
\mathrm{M} \\
\mathrm{FB}+, \mathrm{FZ}+\mathrm{H}, \mathrm{MB}+, \mathrm{MZ}+\mathrm{H} \\
\mathrm{FZ}+, \mathrm{FB}+\mathrm{W}, \mathrm{MZ}+, \mathrm{MB}+\mathrm{W} \\
\mathrm{FB}-, \mathrm{FZ}-\mathrm{H} \\
\mathrm{FZ}-, \mathrm{FB}-\mathrm{W} \\
\mathrm{MB}-, \mathrm{MZ}-\mathrm{H} \\
\mathrm{MZ}-, \mathrm{MB}-\mathrm{W} \\
\mathrm{HF} \\
\mathrm{HM} \\
\text { WF } \\
\text { WM }\end{array}$ & $\begin{array}{l}\text { pho: } \\
\text { mae: } \\
\text { lung } \\
\text { pa: } \\
\text { 'a:w } \\
\text { 'a: } \\
\text { na: (ba:w) } \\
\text { na: (sa:w) } \\
\text { pho: pu: } \\
\text { mae: ja: } \\
\text { pho: jai/pho: sô:n } \\
\text { mae: jai/mae: sô:n }\end{array}$ \\
\hline $\begin{array}{c}\mathrm{G} 0 \\
\text { (Ego non marié) }\end{array}$ & $\begin{array}{l}\mathrm{B}+ \\
\mathrm{Z}+ \\
\mathrm{B}- \\
\mathrm{Z}- \\
\mathrm{Z}+\mathrm{H} \\
\mathrm{B}+\mathrm{W} \\
\mathrm{Z}-\mathrm{H} \\
\mathrm{B}-\mathrm{W} \\
\mathrm{F} / \mathrm{MB}+/ \mathrm{Z}+\mathrm{S} \\
\mathrm{F} / \mathrm{MB}+/ \mathrm{Z}+\mathrm{D} \\
\mathrm{FB}-/ \mathrm{Z}-\mathrm{S} \\
\mathrm{FB}-/ \mathrm{Z}-\mathrm{D} \\
\mathrm{MB}-/ \mathrm{Z}-\mathrm{S} \\
\mathrm{MB}-/ \mathrm{Z}-\mathrm{D}\end{array}$ & $\begin{array}{l}\text { 'a:j } \\
\text { 'ü:aj } \\
\text { no:ng (sa:j) } \\
\text { no:ng (sa:w) } \\
\text { phi: 'a:j } \\
\text { phi: na:ng } \\
\text { no:ng khoe:j } \\
\text { no:ng phai/no:ng saphai } \\
\text { lung } \\
\text { pa: } \\
\text { 'a:w } \\
\text { 'a: } \\
\text { na: (ba:w) } \\
\text { na: (sa:w) }\end{array}$ \\
\hline $\begin{array}{c}\text { G0 } \\
\text { (Ego marié) }\end{array}$ & $\begin{array}{l}H \\
W \\
\text { SWF } \\
\text { SWM } \\
\text { DHF } \\
\text { DHM } \\
B+, Z+H \\
Z+, B+W \\
B-, Z-H m \\
B-, Z-H W \\
Z-, B-W m \\
Z-, B-W W\end{array}$ & $\begin{array}{l}\text { phwa } \\
\text { mi:a } \\
\text { pho: jai/pho: sô:n } \\
\text { mae: jai/mae: sô:n } \\
\text { pho: pu: } \\
\text { mae: ja: } \\
\text { lung } \\
\text { pa: } \\
\text { 'a:w } \\
\text { na: (ba:w) } \\
\text { 'a: } \\
\text { na: (sa:w) }\end{array}$ \\
\hline $\begin{array}{c}\mathrm{G}-1 \\
\text { (Ego non marié) }\end{array}$ & $\begin{array}{l}\mathrm{B}+/ \mathrm{B}-\mathrm{S}, \mathrm{Z}+/ \mathrm{Z}-\mathrm{S} \\
\mathrm{B}+/ \mathrm{B}-\mathrm{D}, \mathrm{Z}+/ \mathrm{Z}-\mathrm{D} \\
\mathrm{B}+/ \mathrm{B}-\mathrm{DH}, \mathrm{Z}+/ \mathrm{Z}-\mathrm{DH} \\
\mathrm{B}+/ \mathrm{B}-\mathrm{SW}, \mathrm{Z}+/ \mathrm{Z}-\mathrm{SW}\end{array}$ & $\begin{array}{l}\text { la:n (sa:j) } \\
\text { la:n (sa:w) } \\
\text { la:n khoe:j } \\
\text { la:n phai/la:n saphai }\end{array}$ \\
\hline
\end{tabular}




\begin{tabular}{|c|c|c|c|c|}
\hline $\begin{array}{c}\mathrm{G}-1 \\
\text { (Ego marié) }\end{array}$ & $\begin{array}{l}\mathrm{S} \\
\mathrm{D} \\
\mathrm{DH} \\
\mathrm{SW} \\
\mathrm{B}+/ Z+\mathrm{Z} \\
\mathrm{B}+/ Z+\mathrm{D} \\
\mathrm{B}-/ \mathrm{Z}-\mathrm{Sm} \\
\mathrm{B}-/ \mathrm{Z}-\mathrm{Sw} \\
\mathrm{B}-/ \mathrm{Z}-\mathrm{Dm} \\
\mathrm{B}-/ \mathrm{Z}-\mathrm{Dw}\end{array}$ & \multicolumn{3}{|c|}{$\begin{array}{l}\text { lu:k (sa:j) } \\
\text { lu:k (sa:w) } \\
\text { lu:k khoe:j } \\
\text { lu:k phai/lu:k saphai } \\
\text { lung } \\
\text { pa: } \\
\text { na: (ba:w) } \\
\text { 'a:w } \\
\text { na: (sa:w) } \\
\text { 'a: }\end{array}$} \\
\hline$G-2$ & $\begin{array}{l}\text { SS, DS } \\
\text { DD, SD } \\
\text { SDH } \\
\text { SDW }\end{array}$ & \multicolumn{3}{|c|}{$\begin{array}{l}\text { la:n (sa:j) } \\
\text { la:n (sa:w) } \\
\text { la:n khoe:j } \\
\text { la:n phai/la:n saphai }\end{array}$} \\
\hline$G-3$ & $\begin{array}{l}\text { S/DS/DS } \\
\text { S/DS/DD }\end{array}$ & \multicolumn{3}{|c|}{$\begin{array}{l}\text { le:n (sa:j) } \\
\text { le:n (sa:w) }\end{array}$} \\
\hline$G-4$ & $\begin{array}{l}\text { S/DS/DS/DS } \\
\text { S/DS/DS/DD }\end{array}$ & \multicolumn{3}{|c|}{$\begin{array}{l}l o: n(\mathrm{sa}: \mathrm{j}) \\
\operatorname{lo:n}(\mathrm{sa}: \mathrm{w})\end{array}$} \\
\hline & $\begin{array}{l}\mathrm{F}=\text { Father } \\
\mathrm{M}=\text { Mother } \\
\mathrm{B}=\text { Brother } \\
\mathrm{Z}=\text { Sister }\end{array}$ & $\begin{array}{l}S=\text { Son } \\
D=\text { Daughter } \\
H=\text { Husband } \\
W=\text { Wife }\end{array}$ & $\begin{array}{l}\mathrm{m} \\
\mathrm{w} \\
+ \\
\overline{\mathrm{F}} / \mathrm{M}\end{array}$ & $\begin{array}{l}=\text { man speaking } \\
=\text { woman speaking } \\
=\text { older } \\
=\text { younger } \\
=\text { Father or Mother }\end{array}$ \\
\hline & \multicolumn{4}{|c|}{$\begin{array}{l}\text { pho: uniquement attesté chez les Isan } \\
\text { pho: attesté chez les Isan et les Lao } \\
\text { pho: attesté chez les Isan et les Thaï } \\
\text { pho: attesté chez les Isan, les Lao et les Thaï }\end{array}$} \\
\hline
\end{tabular}

Tabl. 1. Termes de parenté isan

dans le système de parenté lao, seul le principe de séniorité est retenu au niveau des catégories classificatoires que représentent les sù:a, et par le jeu des oppositions symboliques jai/no:j (" grand/petit ») ou 'a:j/no:ng («frère aîné/ cadet ») les Lao du nord-est de la Thailande, comme ceux de la rive gauche du Mékong (Condominas $1961: 3$ ), établissent une distinction fondamentale entre les branches issues du mariage des germains aînés du père ou de la mère, les sù:a jai ou sù:a 'a:j et celles qui, résultant du mariage de leurs cadets, sont appelées sù:a no:j ou sù:a no:ng.

Phénomène tout aussi caractéristique de la terminologie lao en usage chez les Isan (et qui devrait être vérifiée dans le cas des Lao établis sur la rive gauche du Mékong), le même découpage de la parentèle en branches aînées et cadettes est reproduit à partir de G0 dès lors qu'Ego se marie. En effet, alors que dans le système siamois le mariage d'Ego n'entraîne aucune modification dans la nomenclature de parenté, chez les Isan le changement de statut marital de ce dernier a des répercussions directes sur les termes qu'il emploie pour désigner 
$\mathrm{G}+2$

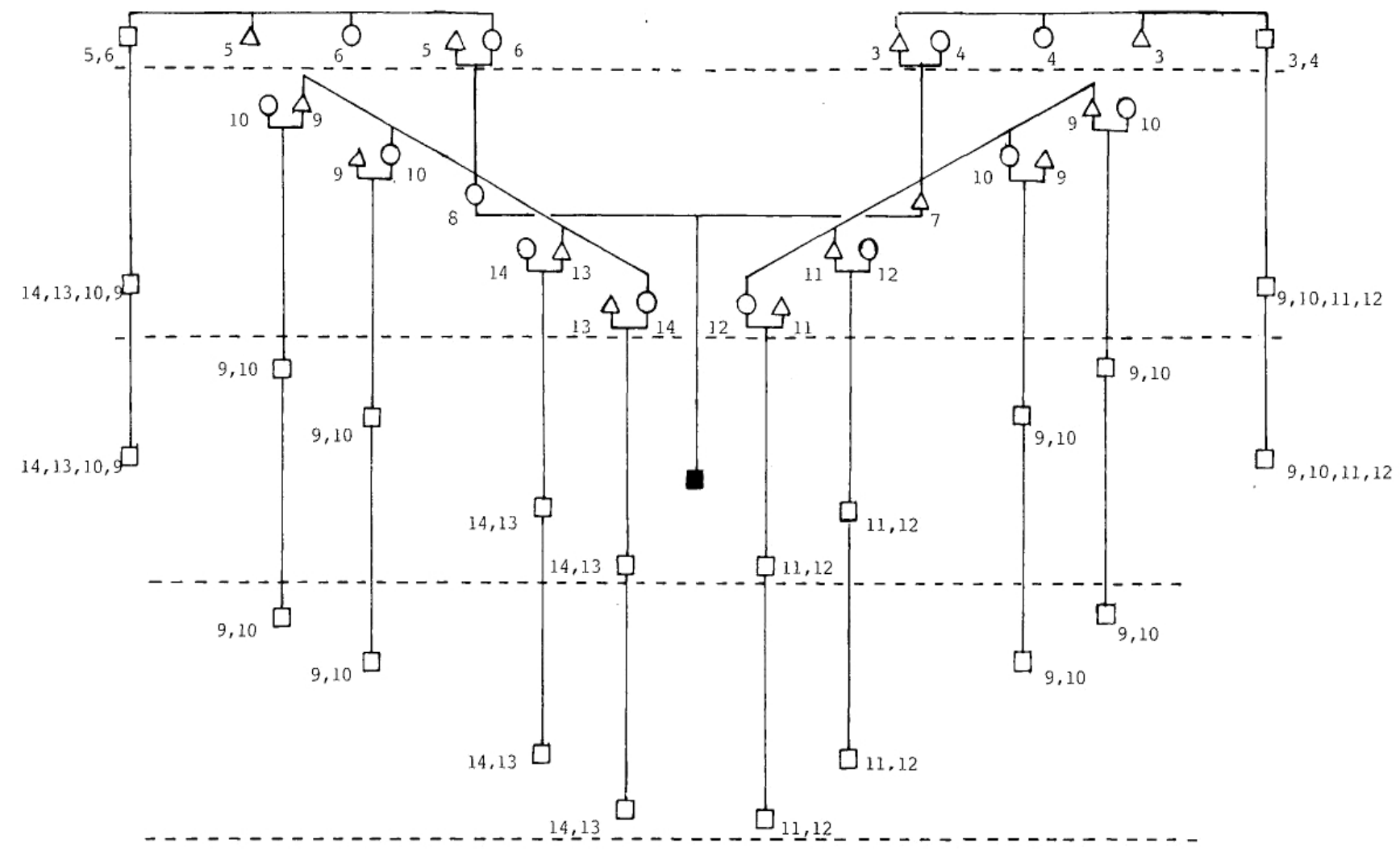

Fig. 1. Terminologie de référence lao

(lignes collatérales définies à partir de $G+2$ et $G+1$ ).

3. pho: pu:

4. mae: ja:

5. pho: jai ou pho: sô:n

6. mae: jai ou mae: sô:n

7. pho:

8. mae:
9. lung

10. pa:

11. 'a:w

12. 'a:

13. na: ba:w

14. na: sa:w 


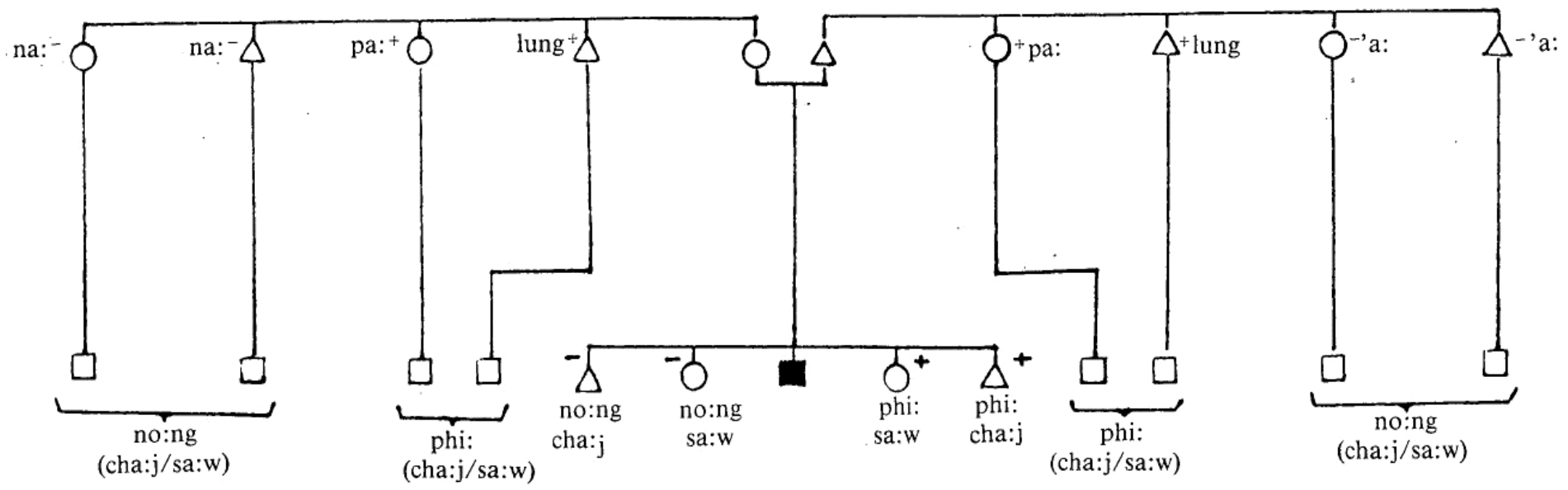

Fig. 2. Termes de référence siamois pour désigner les cousins au premier degré. 
ses germains et leurs conjoints. Célibataire, Ego distingue ses cadets de ses aînés, les premiers étant appelés no:ng avec spécification secondaire du sexe par ajout des déterminants $s a: j$ (marque du masculin) ou $s a: w$ (marque du féminin), les seconds étant d'emblée distingués en fonction de leur sexe grâce à l'opposition 'a:j (frère aîné)/'ù:aj (sœur aînée). Toujours en tant que célibataire Ego distingue systématiquement ses consanguins et ses alliés : le mari de sa sœur aînée est appelé phi: 'a:j, celui de sa sœur cadette no:ng khoe:j, l'épouse de son frère aîné phi: nang, celle de son frère cadet no:ng phai (fig. 3). Après son mariage la terminologie qu'utilise Ego traduit un changement de perspective radical. En effet il se place désormais dans la position de ses enfants nés ou à naître et applique à ses propres frères et sœurs ou à ceux de son conjoint, ainsi qu'à leurs alliés, la taxinomie qu'il emploie habituellement pour désigner ses oncles et tantes patri- ou matrilatéraux.

Le passage du statut de célibataire à celui d'homme ou de femme marié(e)s

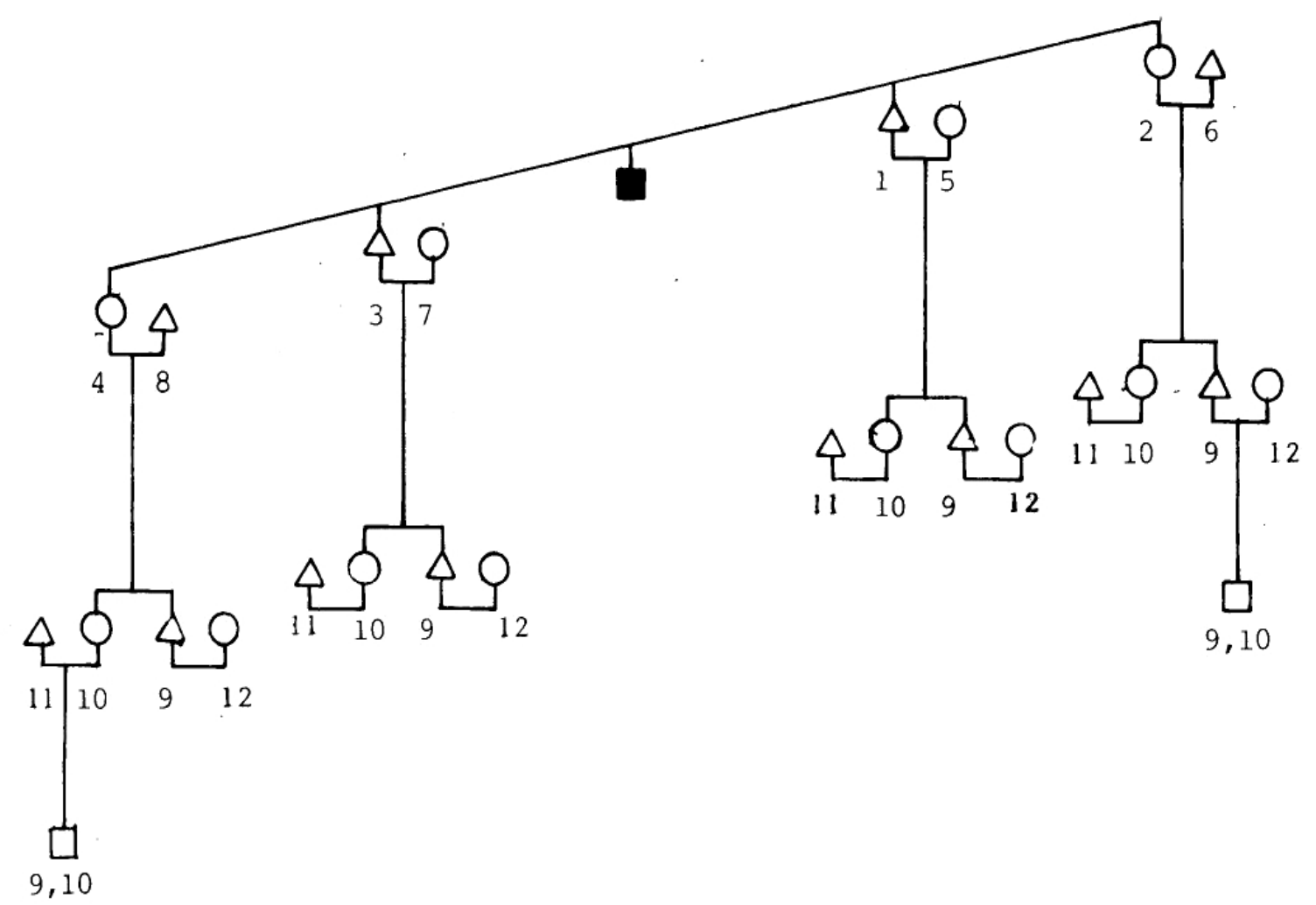

Fig. 3. Mode de désignation des germains et des enfants de germains

(Ego célibataire).
1. 'a:j
2. 'ù:aj
7. no:ng phai
3. no:ng sa:j
8. no:ng khoe:j
4. no:ng sa:w
9. la:n sa:j
5. phi: nang
10. la:n sa:w
6. phi: 'a:j
11. la:n phai
12. la:n khoej 
a également des répercussions sur la terminologie appliquée aux enfants de germains ou à leurs conjoints. Alors qu'avant le mariage Ego désigne cette catégorie de collatéraux par le terme la: $n$ assorti subsidiairement d'un déterminant (sa:j pour un consanguin de sexe masculin, sa: $w$ pour son équivalent de sexe féminin, khoe:j pour un allié, phai pour une alliée) (fig. 3), une fois marié il établit une équivalence formelle entre ses germains directs et leurs enfants, définissant ainsi à partir de G0 des branches aînées et cadettes identiques à celles qu'il délimite à partir de $\mathrm{G}+1$ lorsqu'il assimile ses oncles ou tantes à leurs descendants (fig. 4 \& 5).

Si à partir de G0 la définition des sù:a dépend du statut marital d'Ego et du changement de perspective qui s'ensuit, on note d'une manière générale que la distinction entre branches ne résulte jamais directement de la position structurale qu'occupe Ego dans le champ de la parenté, mais toujours de celle qu'occupe un consanguin situé à un niveau générationnel différent. Nous l'avons vu, Ego doit faire référence à l'ordre de naissance des collatéraux situés à la génération immédiatement supérieure à la sienne pour définir le statut du sù:a auquel il appartient, alors qu'en retour il n'est en droit d'inscrire sa génération et les suivantes à l'intérieur de branches collatérales que lorsque sa des-

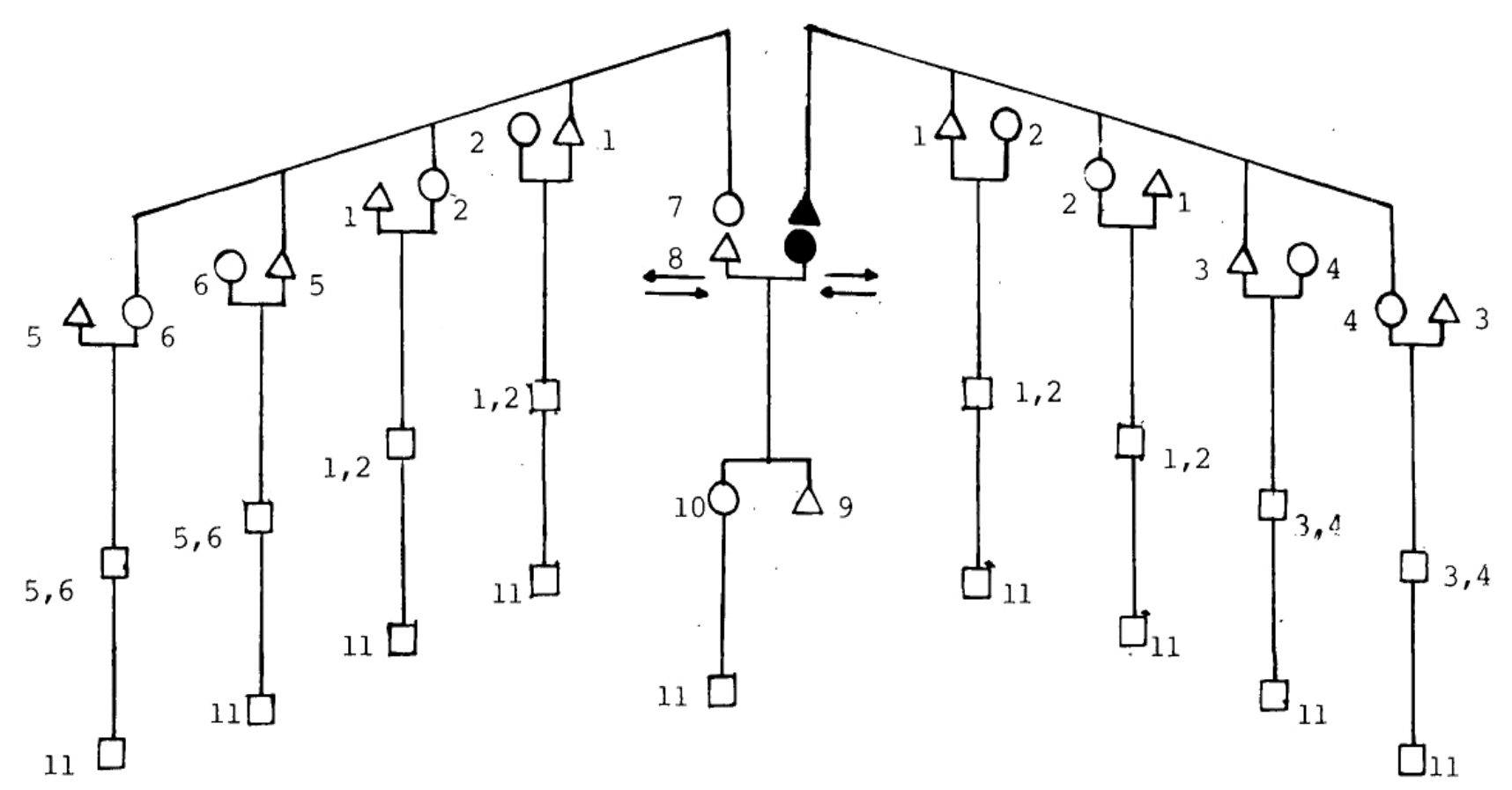

Fig. 4. Mode de désignation des germains et de leurs descendants

1. lung

2. pa:

3. 'a:w

4. 'a:

5. na: ba:w

6. na: sa:w

(Ego marié).

7. mi:a

8. phwa

9. lu:k sa:j

10. lu:k sa:w

11. la:n 


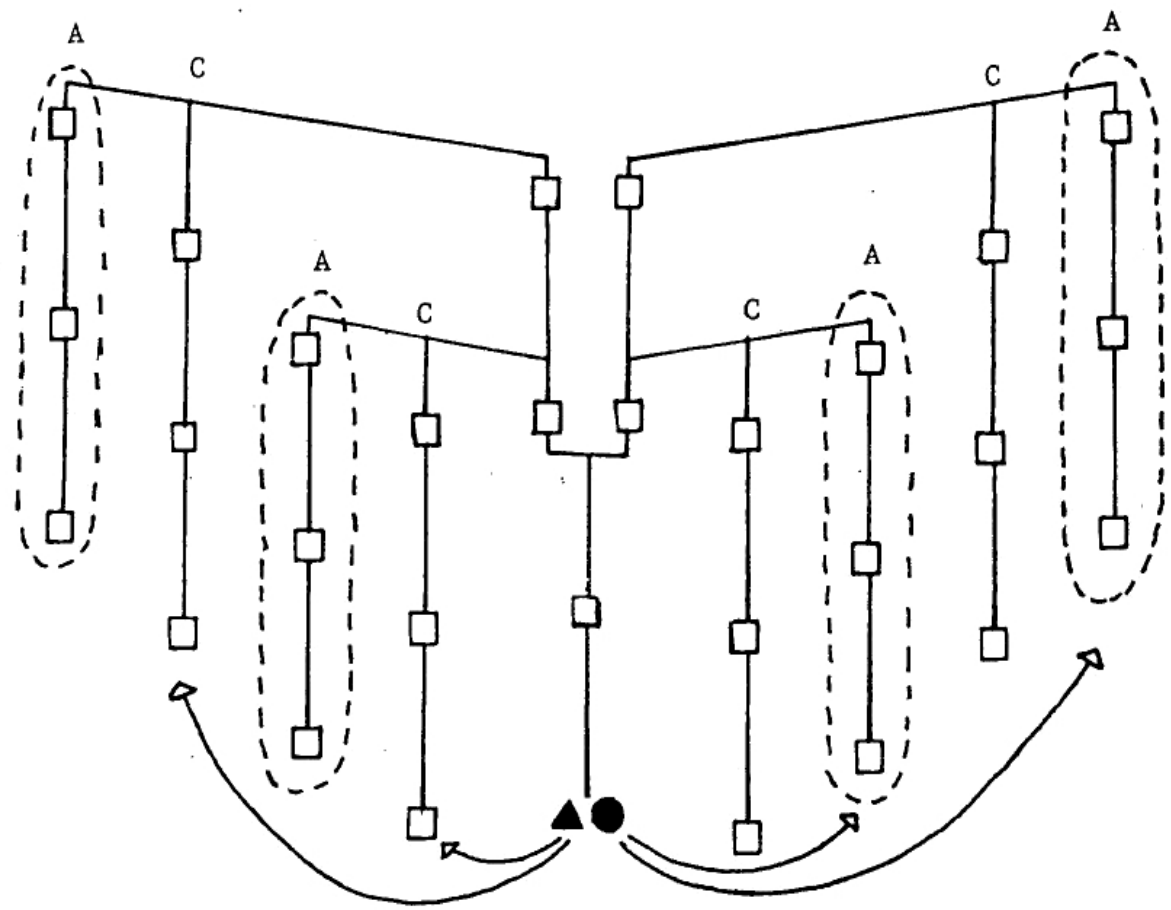
$A$ = ligne aînée
$C=$ ligne cadette
$\leftarrow=$ mariage possible

Fig. 5. Catégories de cousins épousables pour Ego (selon son sexe).

cendance étant assurée ou postulée en vertu de l'acte de mariage, il est luimême fondateur d'un sù:a et peut dès lors adopter la perspective de ses enfants.

En résumé donc, la relation de dépendance intergénérationnelle sur laquelle se fonde la définition terminologique des sù: $a$ peut être formulée ainsi : la création d'une branche collatérale par Ego dépend de l'existence de ses enfants, que celle-ci soit réelle ou institutionnellement possible, alors qu'en retour le statut relatif du sù:a auquel ces enfants appartiennent dépend du statut d'Ego.

Les remarques qui précèdent suggèrent d'emblée une question : un même individu peut-il simultanément se réclamer du sù:a fondé par ses parents et de celui qu'il fonde par le truchement de ses enfants ? Une telle question serait pertinente si les lignes définies à chaque génération faisaient office de corporate group et non, comme c'est le cas, de catégories de référence exclusivement utilisées pour définir au sein de la parentèle le champ des choix matrimoniaux offerts à l'individu. Le problème de la double appartenance ou plutôt de la double référence ne se pose donc pas : dès lors que l'individu est marié, son intérêt se déplace logiquement des sù: $a$ définis à partir des générations supé- 
rieures et qui lui spécifiaient les catégories de cousins épousables vers ceux définis à partir de sa génération et qui déterminent les choix matrimoniaux de ses enfants.

\section{CHOIX MATRIMONIAUX ET SU்:A}

Bien qu'un auteur (Doré 1987 : 281-282) fasse mention chez les Lao d'une longue tradition de mariages préférentiels entre cousins parallèles, ce type de mariage n'est attesté que dans la région de Louang Prabang et semble surtout avoir cours dans certains milieux aristocratiques. Chez les Isan cette pratique ne fait pas l'objet de prescriptions et n'est pas citée par nos informateurs comme un idéal à atteindre. De plus, si quelques rares cas peuvent être décelés à l'analyse des généalogies de Ban Amphawan et de Ban Han, ils ne tranchent pas en nombre par rapport aux unions entre cousins croisés et représentent au demeurant un faible pourcentage du total des mariages $(1,5 \%)$. Enfin, indice du peu de signification structurale de cette forme d'alliance, la terminologie lao ne fait, rappelons-le, aucune distinction entre cousins croisés et parallèles. $\mathrm{Si}$ l'on s'en tient aux tendances dominantes, les choix matrimoniaux sont réglés chez les Lao et les Isan non par des prescriptions qui assigneraient à l'individu une classe spécifique de partenaires matrimoniaux, comme c'est le cas dans les systèmes élémentaires, mais à l'inverse par un ensemble de prohibitions clairement définies. Ces prohibitions fonctionnent dans un espace de relations qui ne relève pas exclusivement du domaine de la parenté. Elles portent sur des catégories sociales de référence et non sur des positions structurales définies à l'échelon individuel. De ce fait elles permettent de rattacher les systèmes de parenté lao ou isan aux systèmes semi-complexes que $F$. Héritier (1981: 74) dëcrit en ces termes : "Systèmes de parenté [...] qui signalent, au moyen de prohibitions explicites, les groupes sociaux définis par rapport à Ego ou les classes de consanguins par rapport au même Ego ou à un parent tiers, à l'intérieur desquels il n'est pas possible de choisir son conjoint. "

On ne relève pas de différences entre les Lao des rives droite et gauche du Mékong pour ce qui est des règles négatives concernant le choix du conjoint. Ces prohibitions peuvent être énoncées de la manière suivante :

Règle 1. - Ego ne peut avoir de relations sexuelles et par suite se marier à l'intérieur du sù:a auquel il appartient. Conformément à ce principe d'exogamie sont exclus de ses choix matrimoniaux ses frères et sœurs, parents, grands-parents, enfants et petits-enfants. Cet interdit qui exprime le tabou universel de l'inceste est strictement respecté dans les deux villages étudiés.

Règle 2. - Ego ne peut épouser un membre d'une autre génération que la sienne. En vertu de ce principe il ne peut notamment épouser une personne appartenant à la catégorie des pho: mae: (" pères/mères 》) ou à celle des lu:k la:n (« enfants/petits-enfants »). Les expressions pho: mae: et lu:k la:n servent 
ici de concepts classificatoires grâce auxquels les terminologies de référence ou d'adresse désignent les individus apparentés ou non qui, compte tenu de leur âge, pourraient soit être les géniteurs d'Ego, soit ses enfants ou petits-enfants. Leur âge relatif par rapport à Ego étant en l'occurrence le critère de classification déterminant, position généalogique et affectation générationnelle ne correspondent pas nécessairement. Il se peut en effet que le cumul progressif des écarts d'âge entre collatéraux aînés et cadets fasse qu'un cousin au $2^{\mathrm{e}}$ ou au $3^{\mathrm{e}}$ degré soit classé à un niveau générationnel différent, alors qu'il appartient en réalité au même niveau généalogique qu'Ego.

Règle 3. - Ă G0 le champ des relations de collatéralité se fractionne du point de vue d'Ego : d'une part les cousins au premier degré patri- ou matrilatéraux ; d'autre part l'ensemble extensif qui, à partir des cousins au second degré, dépasse les confins de la mémoire généalogique pour englober au bout du compte tous les célibataires, parents ou non, appartenant à la génération d'Ego. Le premier ensemble est désigné par l'expression classificatoire 'a:j no:ng (" frères aînés/cadets 》). La proximité généalogique de ses membres par rapport à Ego interdit à celui-ci d'établir des relations sexuelles avec eux et de les épouser. Dans la pratique cette interdiction est largement respectée tout comme la précédente (règle 2) et, dans notre échantillon, aucune exception n'est constatée. Quant au second ensemble, identifié par l'expression phi:/no:ng (" aînés/cadets »), il est ouvert aux relations matrimoniales, même si en son sein l'éventail des conjoints possibles se trouve réduit par le jeu des prohibitions qui suivent.

Règle 4. - Au sein de sa propre génération un homme ne peut épouser une femme qui s'inscrit par rapport à lui dans la catégorie des aînés. Il faut en effet que l'inégalité statutaire de base entre hommes et femmes que postule l'idéologie soit renforcée et non pas contredite par la relation hiérarchique entre aînés (phi:) et cadets (no:ng), le statut des premiers étant supérieur à celui des seconds. Le non-respect de ce principe de concordance statutaire est perçu comme un facteur déstabilisant : il n'est ni convenable d'un point de vue moral (sadwak), ni faste (monkhon) pour le destin du futur ménage. Dans les villages étudiés ce principe n'est transgressé que dans un petit nombre de cas (12 sur 156, soit 7,6 \% à Ban Amphawan ; 6 sur 268 soit 2,2 \% à Ban Han). L'écart qui sépare l'âge des conjoints est égal ou inférieur à 2 ans pour $37,6 \%$ des couples, égal ou supérieur à 5 ans pour 72,8\% d'entre eux et ne dépasse 10 ans que dans $10 \%$ des cas, l'écart maximal étant de 17 ans. On note d'autre part qu'au sein des couples qui ont opté pour cette solution "atypique " les conjoints entretiennent alors un rapport fictif aîné/cadet, rapport que symbolise, conformément à l'usage, l'emploi par l'épouse du terme phi: à l'adresse de son mari et en retour l'usage par celui-ci du terme no:ng à l'adresse de sa femme.

Règle 5. - Elle proscrit le mariage de deux cousins lorsque le garçon appar- 
tient à une branche cadette (sù:a no:j) et la fille à une branche aînée (sù:a jai). Visant comme la précédente à assurer une concordance statutaire entre le sexe et l'ordre de naissance, cette règle présente la double caractéristique d'opérer à l'intérieur de la parentèle et d'appliquer le critère sélectif de l'âge non pas aux conjoints, mais aux fondateurs des branches collatérales dans lesquelles ils s'inscrivent. Exclusivement fonction du rapport aîné/cadet entre collatéraux directs de générations supérieures, elle oblitère le principe de la séniorité du mari qui est en vigueur dans un contexte plus général (règle 4). Il est admis en effet qu'un homme épouse une cousine plus âgée que lui dès lors que celle-ci appartient à une branche cadette. La substitution d'un interdit à un autre confère par conséquent aux choix matrimoniaux internes et externes à la parentèle une latitude sensiblement équivalente.

La règle 5 exprime à l'évidence deux principes structuraux sous-jacents : d'une part les positions statutaires résultant de l'ordre de naissance des consanguins déterminent au sein de la parentèle ceux définis par l'alliance ; d'autre part le statut relatif des collatéraux ascendants détermine celui des descendants. Logiquement, grâce à l'articulation de ces deux principes, l'interdit matrimonial joue d'autant moins lorsque le garçon appartient à une branche aînée et la fille à une branche cadette (fig. 5) que dès lors, en se mariant, les jeunes gens tissent entre leurs sù:a une relation d'affinité qui est statutairement conforme à la relation de consanguinité préétablie entre les aïeuls fondateurs de ces sù:a.

L'interdiction de mariage entre homme d'une branche cadette et femme d'une branche aînée est surtout effective entre cousins au second degré. En deçà opère la règle 3 qui proscrit le mariage d'enfants de germains ; au delà, comme c'est bien souvent le cas en régime indifférencié, la mémoire généalogique s'estompe rapidement et avec elle la référence aux fondateurs des sù:a. Selon leur âge par rapport à Ego les cousins sont dès lors assimilés à des parents, des aînés ou cadets, ou encore à des enfants classificatoires et sont considérés comme tels sur le plan matrimonial. A partir des cousins au troisième degré l'effacement des liens généalogiques donne aux Isan la possibilité d'un rééquilibrage des échanges matrimoniaux asymétriques qui sont de mise entre branches aînées et cadettes, les premières cédant en théorie plus d'époux qu'elles n'en reçoivent. Seul le traitement statistique d'un grand nombre de mariages permettrait de déterminer dans quelle mesure cette possibilité de bouclage est exploitée dans la pratique. A l'échelle très restreinte où l'on se place et même en limitant la perspective aux cousins au second degré, on note d'une part que la terminologie de référence qui répartit les collatéraux en branches aînées et cadettes n'est pas employée par tous ; d'autre part que la portée de l'interdit de mariage avec les cousines de la branche aînée est limitée par de multiples transgressions dont certaines se produisent en chaîne, comme en témoigne l'exemple relevé dans l'un des villages de trois branches collatérales unies entre elles à la seconde génération descendante par des alliances matrimoniales qui expriment une relation statutaire inverse de celle qui existait entre leurs membres fondateurs (fig. 6). 


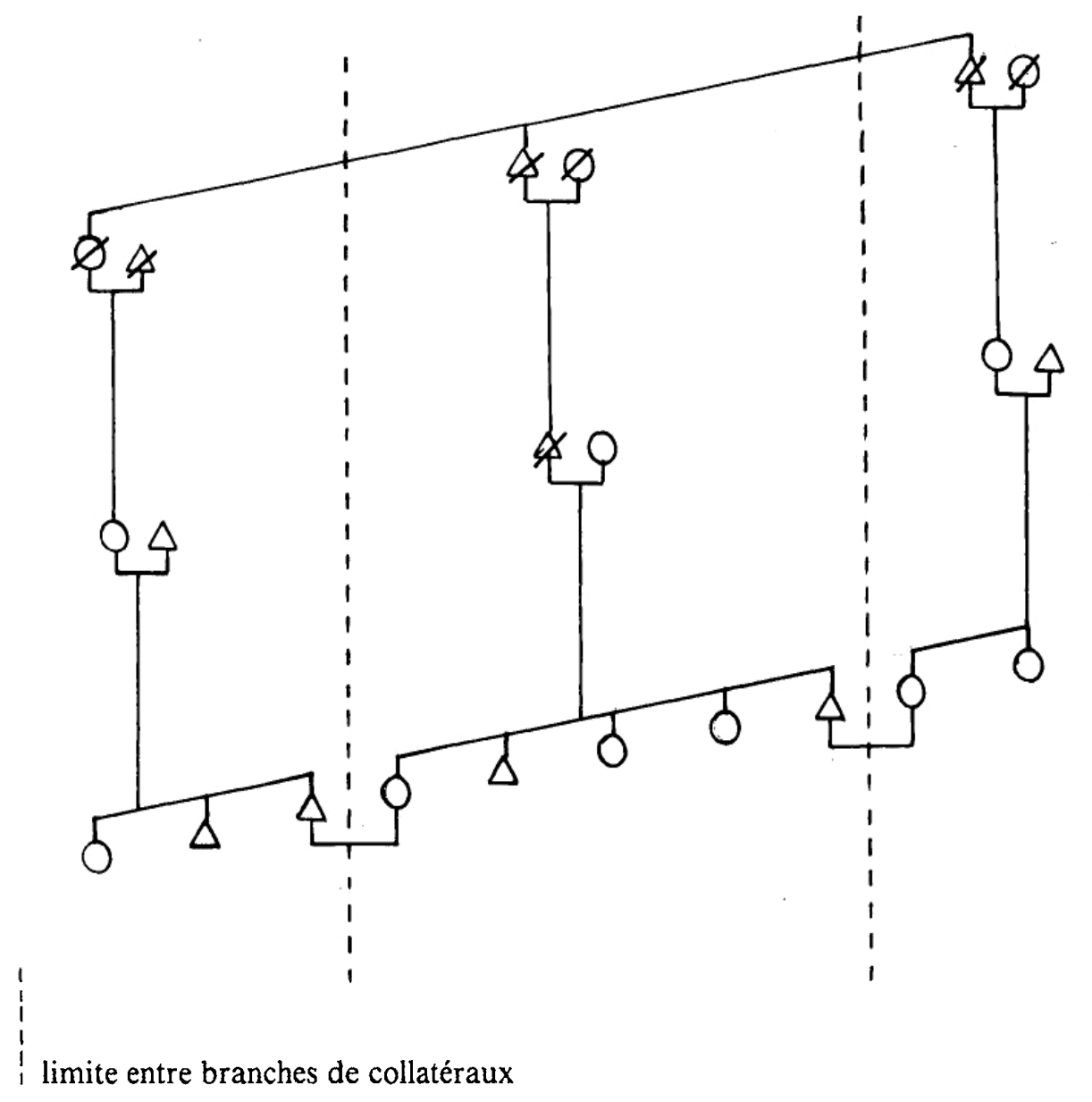

Fig. 6. Cas de transgression en chaîne de la règle de mariage entre branches aînées et cadettes.

On l'a vu précédemment, la terminologie de parenté siamoise fait également dériver le statut des cousins de l'âge relatif des oncles et tantes par rapport aux parents d'Ego. On note cependant que l'équivalence statutaire qui est établie entre collatéraux situés à $\mathrm{G}+1$ et G0 n'a pas d'incidence sur le plan matrimonial. En effet, cette équivalence couvrant uniquement les cousins au premier degré et le mariage avec ceux-ci étant théoriquement exclu selon un interdit analogue à la règle 3 énoncée à propos des Lao, le principe de séniorité est alors masqué et n'intervient pas pour définir des possibilités ou impossibilités de mariages entre lignes de collatéraux.

\section{LES VILLAGES AÎNÉS ET CADETS}

L'étude statistique des mariages intervillageois indique $a$ priori que l'interdit qui règle les relations matrimoniales entre branches collatérales au sein de la parentèle est transposé aux localités situées dans le même voisinage, 
dès lors qu'elles sont investies du statut relatif de $m u$ : ba:n phi: (« village aîné ») et de mu: ba:n no:ng ("village cadet »). Les communautés qui entretiennent ce type de rapport hiérarchique sont unies par un lien généalogique : l'émigration d'une partie de la population du village " aîné » ayant permis la création de celui qualifié de « cadet ».

Si l'engendrement d'une communauté par une autre serait plus fidèlement rendu par l'opposition symbolique pho: mae:/lu:k (" parents/enfants ») que les Lao emploient fréquemment pour signifier la subordination d'une personne ou d'une chose à une autre ${ }^{7}$, le recours à l'opposition phi:/no:ng (" aîné/ cadet ॥) est dû à l'existence de relations matrimoniales intervillageoises. En effet, alors que l'expression phi: no:ng désigne une catégorie de conjoints potentiels, il n'en va pas de même de l'expression pho: mae:lu: $k$ qui symbolise à l'inverse une relation de consanguinité couverte par le tabou de l'inceste.

Dans la mesure où l'interdit qui structure normalement les relations matrimoniales entre aînés et cadets à l'échelle des branches collatérales est effectivement transposé au niveau intervillageois, on doit s'attendre à ce que le village " aîné » cède plus de conjoints de sexe masculin qu'il n'en reçoit de son « cadet » du fait, d'une part, de l'uxorilocalité caractéristique du système de parenté lao et, d'autre part, de la concordance qui doit s'instaurer entre la supériorité statutaire de l'homme et celle de l'aîné. Les données statistiques recueillies dans les deux villages étudiés confirment effectivement cette projection théorique. Ainsi Ban Amphawan, en tant que village cadet, reçoit de son â̂né - une localité voisine située à moins d'l $\mathrm{km}$ - quatre fois plus d'époux qu'il ne lui en donne. Quant à l'autre communauté, Ban Han, la balance des échanges matrimoniaux avec le village voisin qui lui a donné naissance s'établit en sa faveur selon un rapport d'un pour trois. Notons encore que le même type de relation asymétrique est constaté entre Ban Han et ses deux villages satellites, Ban Khok Sahakon situé à $2 \mathrm{~km}$ et Ban Nong Sawan à $1,6 \mathrm{~km}$, à la nuance près que notre communauté de référence étant dès lors placée en position d'aînée, la balance des échanges matrimoniaux s'établit cette fois en sa défaveur : elle cède plus d'époux qu'elle n'en reçoit.

Malgré l'adéquation constatée entre le schéma relationnel aîné-cadet qui structure les alliances au sein de la parenté et la balance des mariages intervillageois, l'hypothèse d'une régulation de ces derniers selon une norme consciente et systématique est fragile. D'abord parce que les Isan nient l'existence d'un interdit qui empêcherait les hommes du village « cadet » de prendre une épouse dans le village " aîné ». Ensuite parce que l'avis que donnent les parents sur les choix matrimoniaux de leurs enfants se fonde seulement, hormis les prohibitions explicites précédemment énoncées, sur la prospérité et la respectabilité des partenaires pressentis. C'est d'ailleurs la mauvaise réputation du village aîné que les habitants de Ban Amphawan et Ban Han mettent en avant pour justifier le déséquilibre des échanges matrimoniaux en leur faveur. Les hommes de leurs communautés sont en effet d'autant moins enclins à prendre une épouse et de ce fait à émigrer dans le $m u$ : $b a: n$ phi: que celui-ci est, selon l'opinion 
courante, peuplé de nak leng (voyous) et est dénué de rabiap (ordre). Le jugement de valeur négatif ainsi porté sur le village " aîné », pour être éminemment subjectif, n'en coïncide pas moins avec un processus politique scientifiquement observable, à savoir que plus la taille d'un village augmente et plus celui-ci perd en cohésion et devient difficile à administrer. La croissance démographique des villages les plus anciennement créés, entraînant un problème foncier et amplifiant l'émigration, peut justifier d'une autre manière le déséquilibre des échanges matrimoniaux entre villages « aînés 》 et « cadets » En effet on peut supposer qu'au moins dans un premier temps le village le plus récent, disposant de terres abondantes non défrichées, ait attiré les jeunes hommes pauvres des villages environnants, notamment de la communauté avec laquelle de solides liens de parenté préexistaient. La faible profondeur historique des localités étudiées, qui ont toutes deux été fondées il y a moins de cent ans, expliquerait que les traces d'un tel transfert de population par voie matrimoniale demeure perceptible sur le plan statistique. Ainsi, que l'on prenne en compte les facteurs d'ordre socio-politique ou économique, c'est moins la supériorité statutaire du village "aîné » que son antériorité historique qui explique l'attraction qu'exerce le village «cadet» sur les jeunes célibataires et l'asymétrie des échanges matrimoniaux observée en sa faveur.

L'objet de cette étude était de mettre en évidence les mécanismes logiques particuliers qui structurent le système de parenté lao. Ces mécanismes tiennent notamment à une règle de projection verticale et généralisée qui reproduit au niveau des générations descendantes les distinctions statutaires et notamment l'opposition aîné/cadet qu'instaure la terminologie entre les parents d'Ego et leurs germains. La transposition de cette règle de $G+1$ à $G 0$ est fonction du statut marital d'Ego et du changement d'appui référentiel qu'implique le passage d'individu mariable à celui d'individu marié. Son champ d'application est donc à géométrie variable. Cependant sa finalité reste toujours la même : délimiter et hiérarchiser, grâce à l'opposition aîné/cadet, des lignes de collatéraux qui, en vertu de leur statut relatif, sont accessibles ou non au mariage. On en vient donc logiquement à la seconde originalité du système de parenté lao. Celui-ci constitue en effet un compromis entre systèmes élémentaires et systèmes complexes d'alliance. Il se rapproche des premiers dans la mesure où il porte non pas sur des individus définis par leur degré de parenté ou leur position généalogique par rapport à Ego, mais sur des groupes ou des catégories sociales. Il s'apparente aux seconds dans la mesure où les catégories de personnes épousables ne sont pas expressément définies à travers l'énoncé de prescriptions, mais sont déductibles de l'exercice de règles négatives.

De par son mode de fonctionnement le système de parenté lao s'inscrit donc dans ce que F. Héritier appelle les structures semi-complexes d'alliance; par là il constitue l'un des rares exemples de la mise en cuvre de ces structures semi- 
complexes dans le cadre de systèmes indifférenciés fonctionnant à l'échelle de vastes espaces sociaux.

L'exemple des deux villages étudiés indique que chez les Lao, comme dans toute autre société, un décalage notable existe entre l'énoncé des règles de parenté et leur mise en pratique. Ce décalage, variable suivant la nature des règles en jeu et la personnalité des acteurs sociaux, est particulièrement accentué au niveau tant de la détermination des branches collatérales que des prohibitions matrimoniales qui leur sont associées. Dès lors une question se pose : un tel phénomène est-il généralisable aux Lao des deux rives du Mékong ou bien est-il relatif aux seuls Isan ? Auquel cas on pourrait y voir l'effet d'une influence du système de parenté siamois où ces principes structuraux ne jouent pas. En l'état actuel, la réponse à cette question dépend d'une comparaison qui reste à établir entre les usages sociaux que les Lao des deux rives du Mékong font de la parenté.

Ayant évolué parallèlement à partir d'un même fond culturel, celui des groupes de langue thaï, les systèmes de parenté lao et siamois ont logiquement de nombreux points en commun. De type indifférencié, ils présentent l'un et l'autre une inflexion matrilinéaire tant sur le plan successoral que dans le choix du mode de résidence des jeunes mariés. Ils réduisent d'autre part le champ des possibilités matrimoniales au moyen de prohibitions dont certaines sont communes aux deux systèmes (interdiction d'épouser un cousin au premier degré ou pour un homme de se marier avec une femme plus âgée que lui). Les activités de production et les formes d'entraide s'organisent principalement à l'intérieur de familles mono- ou polynucléaires qui forment en l'occurrence l'unité sociale de base. De plus les comportements associés aux rapports entre consanguins généalogiquement proches servent de modèle idéal aux rapports hiérarchiques de type patron-client qui paraissent structuralement prépondérants dans un contexte social plus large.

En dépit de leurs points communs, ces deux systèmes de parenté divergent néanmoins par la manière dont ils intègrent le critère de la séniorité comme principe structurant. Le mécanisme de transfert d'une génération sur l'autre des positions structurales définies par l'âge correspond dans le système siamois non pas à une règle de projection verticale vers le bas des termes de parenté, comme c'est le cas chez les Lao, mais à une règle de projection horizontale. Le champ d'action de ce mécanisme de transfert est d'autre part beaucoup plus limité dans le système siamois puisqu'il opère entre deux générations seulement (entre $\mathrm{G}+1$ et $\mathrm{G} 0$ ). Il ne prête pas de ce fait à la formation de catégories classificatoires analogues aux sù: $a$ lao et ne sert pas d'élément régulateur sur le plan matrimonial. Conséquemment, on a affaire avec l'exemple siamois à un système complexe d'alliance.

D'une architecture typiquement lao tout en étant teinté d'emprunts au vocabulaire siamois, le système de parenté isan présente, avec celui des Lao Yüan, la particularité d'admettre des groupes de filiation fondés sur le culte d'un ancêtre maternel commun et dont la finalité est strictement religieuse. 
Doit-on voir dans l'existence de tels groupes le vestige d'une forme d'organisation jadis commune aux rives droite et gauche du Mékong, ou bien le produit d'une empreinte culturelle externe qui se serait imposée à la même époque ou successivement aux seules ethnies Yüan et Isan. Si la persistance d'un cycle annuel de rites chez les Yüan et l'existence dans cette ethnie de médiateurs attitrés auprès du génie ancestral témoignent, semble-t-il, d'un enracinement plus profond de cette forme de culte et laissent supposer sa diffusion aux Isan par l'intermédiaire des Yüan, le manque de données historiques relatives à ce type de croyance ne permet cependant pas de trancher. Quelle que soit son origine, on note que la croyance en un ancêtre maternel et les groupes de filiation qui lui sont associés connaissent aujourd'hui un sensible déclin dans les deux ethnies considérées, ce déclin paraissant plus accentué chez les Isan.

Département d'Ethnologie, Université Paris $X$, Nanterre

NOTES

* Cette étude, financée, pour partie par le CNRS (ER 300), pour partie par le Ministère des Affaires étrangères, a été menée lors de deux missions que j'ai effectuées en Thailande : la première de septembre 1984 à mars 1986 ; la seconde de février à mai 1988 . Je tiens d'autre part à remercier Mme F. Héritier-Augé dont les précieuses remarques ont favorisé la réalisation de cette étude.

1. La transcription en caractères latins des noms de lieux est conforme à celle de l'administration thailandaise. La transcription des noms de groupes ethniques correspond à l'usage courant. Le système de transcription des termes isan utilisé ici se veut le plus simple et le plus proche possible du mode de prononciation vernaculaire. Il s'établit comme suit : les consonnes aspirées $k, t$ et $p$ sont écrites avec un $h$ lorsqu'elles sont placées à l'initiale d'un mot, seule position où l'aspiration est marquée ; ' indique l'arrêt glottal ; la consonne thaï $\varepsilon$ est indiquée par $j$ qui a la même valeur phonétique que le y français lorsqu'il fait office de consonne ; : placé après une voyelle indique que celle-ci est longue ; les caractères $\hat{o}$ et $o$ indiquent respectivement un o fermé et un o ouvert; le e ouvert est indiqué par ae; et le $\dot{u}$ et le oe correspondent respectivement au $y$ et au $\partial$ du système API ; la diphtongue $a o$ correspond à la voyelle thaí _ ; les caractères thaï $Y$ et $q$ sont rendus par la diphtongue ai qui doit être prononcée comme le français « ail ». Bien que le thaï, le lao et l'isan soient des langues à tons, on a pris le parti de ne pas marquer ceux-ci afin de ne pas compliquer le système graphique choisi.

2. Bien qu'il ne fasse pas l'unanimité parmi les ethnologues le concept de ramage, proposé par R. Firth, a le mérite de préciser sans équivoque la nature indifférenciée du système de parenté à propos duquel il est employé.

3. Le cycle de la maisonnée débute lorsque le jeune couple a économisé assez d'argent pour construire sa propre maison. Cet acte ne signifie pas pour autant que le couple soit totalement autonome. Il le devient le jour où il obtient soit par héritage, soit par achat une parcelle de rizière qu'il exploitera pour son propre compte. Entre-temps il peut rester plusieurs années partiellement dépendant des parents de l'un ou l'autre des conjoints. La formule isan qui est adaptée à cette situation de dépendance est la suivante : het kan, bae:ng kha:w tae kin khon la bon, sai ngoen khon la thung (" on travaille ensemble, on partage le riz, mais on mange en des endroits différents et on place l'argent dans des bourses séparées "). Le temps passant, le ménage qui a accédé à l'autonomie résidentielle s'accroît au fil des naissances. Il fonde sa propre exploitation et devient économiquement autonome. Ce stade est défini par la formule : pho: mae: li:ang lu:k (« les parents élèvent les enfants »). Le temps s'écoule encore et le ménage marie son premier enfant. Selon la tradition, le jeune couple vit auprès des parents de l'épouse. La maisonnée bénéficiant de l'apport de main-d'œuvre fournie par le gendre, la taille de l'exploitation et sa propriété tendent à augmenter. Les Isan décrivent une telle situation de la manière suivante : het le kin namkan, ju: bon diawkan (« on travaille et on 
mange ensemble, on habite ensemble $\gg$ ). Dans un stade ultérieur, le couple fondateur de la maisonnée atteint un âge qui ne lui permet plus de travailler sur l'exploitation. Il en confie la gestion au dernier enfant marié qui vit auprès de lui. Désormais, de soutien de famille il devient assisté. La formule employée est alors : kin namkan, lu:k li:ang pho: mae: ( on mange ensemble, les enfants nourrissent les parents $»)$.

4. Si ces notions sont connues des paysans isan, elles sont, comme le remarque KeMP (1984:66), rarement employées dans le discours quotidien, ce qui tendrait à prouver que les normes qu'elles recouvrent sont peu souvent transgressées et donc puissamment intériorisées.

5. Le terme tao kae: prend plusieurs sens en thaï contemporain. Il désigne : 1) l'intercesseur dans l'arrangement des mariages ; 2) un chinois fortuné (HAAs 1964 : 161) ; 3), un commerçant, entrepreneur ou contractant d'origine chinoise (ThIENGBuRANATHum $1985: 466$ ). Dans le langage courant il prend tout simplement le sens d' « employeur », de " patron ", avec il est vrai une forte connotation ethnique du fait de l'hégémonie des Chinois dans les secteurs industriel, financier et commercial en Thailande et plus généralement en Asie du Sud-Est. Une telle polysémie est particulièrement intéressante. Elle témoigne en effet du télescopage d'un concept indigène, tâo kàe:, qui exprime une charge honorifique liée au statut d' " ancien », et d'un concept allogène, plus précisément teo chiu (dialecte des provinces du Kwangtung et du Fukien), qui se prononçait avant sa thaisation tâo kĕ et qui désigne une fonction économique : celle d'entrepreneur. Cette confusion n'est pas le fait du hasard. Elle traduit la volonté de poser dans les termes d'une relation statutaire inscrite dans la tradition thaï et qui est de ce fait culturellement signifiante et politiquement acceptable, un secteur d'activités que les Chinois ont largement contribué à développer et qu'ils continuent à dominer.

6. Selon REINHORN (1970: 796) le concept de sù:a peut être traduit en français par plusieurs termes qui renvoient soit à une origine commune, soit au groupe qui en résulte. Il peut ainsi signifier tour à tour : "source », « souche », « germe », « espèce », « clan », « famille », " lignée » ou « lignage ». Dans l'usage courant les Isan mettent très souvent en avant le principe de filiation inhérent à ce type de groupe en ajoutant à sù:a le substantif sa:j qui signifie "ligne ", " fil », " chaîne » (ibid. : 577). Les sù:a doivent être distingués des sù:a phi:. En effet, alors que les premiers n'existent que comme catégories classificatoires, les seconds ont une plus grande profondeur généalogique, remplissent une fonction religieuse et prennent corps à l'occasion de certains rites, comme nous l'avons vu précédemment.

7. Par exemple les Lao distinguent la serrure des clefs en appelant la première mae: kuncae: (" mère/clefs ») et les secondes lu:k kuncae: (" enfant/clefs "); ils distinguent le fleuve et ses affluents en opposant mae: nam (« mère/eau ») et lu: $k$ nam (« enfant/eau »), l'employeur et ses employés en nommant le premier pho: ca:ng ("père/louer »), les seconds lu:k ca:ng (« enfant/louer»), ou encore le commerçant et ses clients, le premier étant appelé pho: kha: (« père/vendre »), les seconds lu:k kha: (« enfant/vendre »).

\section{B I B LIO G R A P H I E}

BARBER, Martin John P.

1979 Migrants and Modernization. A Study of Change in Lao Society. London, University of Hull, Ph. D. Thesis.

Cohen, Paul T.\& Gehan Wijeyewardene

1984 «Introduction », Mankind 14 (4), Special Issue $3: 249-262$. Sidney.

Condominas, Georges

1961 «Structure de la famille lao », communication présentée au X $\mathrm{X}^{\mathrm{e}}$ Congrès des Sciences du Pacifique, Honolulu (4 p. ronéo.).

Davrs, Richard

1984 Muang Metaphysics. Bangkok, Pandora Press.

De Young, John

1955 Village Life in Modern Thailand. Berkeley, University of California Press. 
DORÉ, Pierre-Sylvain

1972 De l'Hibiscus à la frangipane. Thèse de $3^{\mathrm{e}}$ cycle. Paris, Université Paris V.

1987 Aux Sources de la civilisation lao. Thèse d'État. Paris, Université Paris V.

HaAs, Mary

1964 Thai-English Student's Dictionary. Stanford, Stanford University Press.

HÉRITIER, Françoise

1981 L'Exercice de la parenté. Paris, Hautes Études / Gallimard / Le Seuil.

IRVINE, Walter

1984 «Decline of Village Spirit Cults and Growth of Urban Spirit Mediumship : the Persistence of Spirit Beliefs, the Position of Women and Modernization ", Mankind 14 (4), Special Issue 3 : 315-324. Sidney.

KaufMaN, Howard Keva

1976 Bangkhuad, a Community Study in Thailand. Rutland/Tokyo, Charles E. Tuttle Comp. (1re éd. 1960.)

KEMP, Jeremy

1970 «Initial Marriage Residence in Rural Thailand », in T. BunnaG \& M. Smrthies, eds., In Memoriam Phya Anuman Rajadhon. Bangkok, The Siam Society : 73-85.

1983 «Towards a Comparative Analysis of Thai Kinship », in E. E. BoEsCH, ed., Thai Culture, Report on the Second Thai-European Research Seminar. Saarbruecken, University of the Saar : 27-44.

1984 «The Manipulation of Personal Relations : from Kinship to Patron-Clientage », in Han Ten BRUMmelhurs \& J. H. KeMP, eds., Strategies and Structures in Thai Society. Amsterdam, University of Amsterdam Anthropological-Sociological Centre (« Dept. of South and Southeast Asian Studies » 31) : 55-70.

KEYES, Charles F.

1975 « Kin Groups in a Thai-Lao Community », in W. G. SkInNER \& T. Kirsch, eds., Change and Persistence in Thai Society. Ithaca, Cornell University Press : 274-299.

KORFF Rüdiger

1983 Who Has Power in Bangkok. Bielefied, University of Bielefield Sociology of Development Research Center (« Working Paper » 40).

1986 Bangkok : Urban System and Everyday Life. Saarbrücken, Verlag Breitenbach Publishers.

KuWinPant, Preecha

1984 "Marketing and the Management of Personal Relations in Wang Thong », in Han Ten BRUMMELHUIS \& J. KEMP, eds., Strategies and Structures in Thai Society. Amsterdam, University of Amsterdam Anthropological-Sociological Centre («Dept. of South and Southeast Asian Studies » 31) : 139-152.

LOUNSBURY, Floyd G.

1964 «A Formal Account of the Crow-Omaha Type Kinship Terminologies» in W. H. GoodENOUGH, ed., Exploration in Cultural Anthropology. New York, Mc Graw-Hill : 351-393.

Lux, Thomas

1969 « The Thai-Lao Family System and Domestic Cycle of Northeastern Thailand », Journal of the National Research Council of Thailand 5 (1-4) : 1-17.

Mougne, Christine $M$.

1984 "Spirit Cults and Matrifocality in Northern Thailand : Demographic Perspective ", Mankind 14 (4), Special Issue $3: 300-307$. Sidney.

Mrzuno, Koichi

1971 Social System of Ban Don Daeng Village. Kyoto, Kyoto University (« Center for Southeast Asian Studies, Discussion Paper $\gg 12-22$ ).

National Statistical OfFice of thalland (NSO)

1984 Report on Population Characteristics, the 1984 Survey of Population Change. Bangkok, Office of the Prime Minister. 
Platentus, Henry

1963 The North-East of Thailand : its Problems and Potentialities. Bangkok, National Economic Development Board.

PoTrER, Jack

1976 Thai Peasant Social Structure. Chicago and London, University of Chicago Press.

RabibHadaNa, Akin

1975 "Clientship and Class Structure in the Early Bangkok Period (1782-1873)», in W. G. SKINNER \& T. A. KIRSH, eds., Change and Persistence in Thai Society. Ithaca and London, Cornell University Press : 93-124.

REINHORN, MarC

1970 Dictionnaire laotien-français. Paris, Éd. du CNRS, 2 vol.

RigGs, Fred W.

1966 Thailand: The Modernization of a Bureaucratic Polity. Honolulu, East-West Center Press.

Sifrin, William J.

1966 The Thai Bureaucracy: Institutional Change and Development. Honolulu, East-West Center Press.

TERWIEL, Barend J.

1984 «Formal Structures and Informal Rules : an Historical Perspective on Hierarchy, Bondage and the Patron-Client Relationship ", in Han Ten Brummelhurs \& J. KemP, eds., Strategies and Structures in Thai Society. Amsterdam, University of Amsterdam Anthropological-Sociological Centre (« Dept. of South and Southeast Asian Studies » 31) : 19-38.

Thiengburanathum, Wit

1985 Thai-English Dictionary. Bangkok, Ruamsat.

TuRTon, Andrew

1972 "Matrilineal Descent Groups and Spirit Cults of the Thai-Yuan in Northern Thailand ", Journal of the Siam Society $60: 217-258$.

WIJEYEWARDENE, Gehan

1967 "Some Aspects of Rural Life in Thailand », in T. Sncock, ed., Thailand : Social and Economic Studies in Development. Canberra, Australian National University Press : 65-83. 


\begin{abstract}
A B S T R A C T
Bernard Formoso, Alliance and Seniority : The Case of the Lao in Northeast Thailand. The Lao kinship system is described in comparison with its Siamese counterpart. This sheds light on the considerable importance this system bestows on the senior/junior dichotomy. This dichotomy is then placed within a more general sociocultural context ; and its impact, analyzed on terminology as well as matrimonial practices.
\end{abstract}

\title{
ZUSA M M EN F A S U N G
}

Bernard Formoso, Allianz und Seniorität. Der Fall der Lao im Nord-Osten Thailands. Der Autor beschreibt das Verwandtschaftssystem der Lao und vergleicht es mit dem ihm entsprechenden siamesischen. Dabei zeigt er die große Bedeutung auf, welche dieses System der Unterscheidung älter/jünger beilegt. Er beschreibt den Ort dieser Unterscheidung in einem allgemeineren sozio-kulturellen Zusammenhang und analysiert ihre Wirkung auf die Terminologie und im Bereich der Heiratspraktiken.

\section{RESUMEN}

Bernard Formoso, Alianza y señioridad. El caso de los Lao del noreste de Tailandia. - El autor describe el sistema de parentesco lao, comparándolo a su homólogo siamés. A lo largo de este trabajo pondrá en evidencia la considerable importancia que este sistema confiere a la dicotomía hijo mayor-pequeño. Reemplaza esta dicotomía en un contexto socio cultural mas general y analiza el impacto tanto en el plano de la terminología como en el registro de las prácticas matrimoniales. 